\title{
LAS NUEVAS TEGNOLOGÍAS DE INFORMACIÓN Y COMUNICACIÓN EN LA EFICACIA DE LA FORMACIÓN PROFESIONAL UNIVERSITARIA DEL LICENCIADO EN ADMINISTRACIÓN DE LA UNMSM
}

\author{
THE NEW INFORMATION AND COMMUNICATION TECHNOLOGIES ON THE EFFECTIVENESS \\ OF TRAINING OF UNIVERSITY DEGREE IN BUSINESS ADMINISTRATION FROM THE NATIONAL \\ UNIVERSITY OF SAN MARCOS \\ José Hugo Tezén Campos* \\ jcamping49@yahoo.com
}

[RECEPCIÓN: JULIO DEL 2014/CONFORMIDAD: SETIEMBRE DEL 2014]

\section{RESUMEN}

Para demostrar la contribución de las nuevas tecnologías de información y comunicación en la eficacia de la formación profesional universitaria del Lic. en Administración de la UNMSM, se aplicaron técnicas propias de la investigación científica, como el análisis documental, encuestas e instrumentos orientados a determinar percepciones de los usuarios directos del servicio educativo, en la unidad de análisis, a fin de dar respuesta al problema de investigación planteado como se indica:

¿En qué medida las nuevas tecnologías de información y comunicación contribuyen al logro de la eficacia de la formación profesional universitaria del Lic. en Administración de la UNMSM?

Problema que motivó el objetivo general que se indica:

Determinar el nivel de contribución de las nuevas tecnologías de información y comunicación al logro de la eficacia de la formación profesional universitaria del Lic. en Administración de la UNMSM.

Para lograr el objetivo propuesto, se consideró la metodología siguiente:

a. En primer lugar, se determina la unidad de análisis que para la realización de la investigación es la carrera profesional de Administración de la Universidad Nacional Mayor de San Marcos.

b. Análisis documental del plan de estudio de la carrera profesional y determinación de las plataformas tecnológicas.

c. Aplicación de instrumentos de recolección de datos, a los usuarios directos, a fin de determinar niveles de empleo de las nuevas tecnologías de información y comunicación en la formación profesional del Lic. en Administración de la UNMSM, datos que se procesaron con el software SPSS.

Luego, se procedió al análisis de los resultados obtenidos, con el propósito de dar respuesta al problema planteado, a través de la conjetura de que las nuevas tecnologías de información y comunicación contribuyen al logro de la eficacia de la formación profesional universitaria del Lic. en Administración de la UNMSM, lo que finalmente se comparó con los objetivos propuestos que determinaron su logro.

Palabras claves: Tecnologías de información y comunicación, eficacia, formación profesional universitaria, licenciado en Administración.

\footnotetext{
* Ingeniero Mecánico-Lic. Administración, Magíster en Administración Universitaria, Dr. en Administración, Maestría Ingeniería Hidráulica Computacional (Universidad Nacional Mayor de San Marcos), Docente Universitario a nivel de Pre y Post Grado UNMSM-UNAC.
} 


\section{ABSTRACT}

To demonstrate the contribution of new information and communication technologies on the effectiveness of university training of Administration Degree in San Marcos, own techniques of scientific research and document analysis, surveys and applied instruments to determine perceptions of direct users of educational services in the unit of analysis in order to answer the research question posed as follows:

To what extent the new information and communication technologies contribute to achieving effectiveness of university training of Administration Degree in San Marcos? Problem that motivated the overall objective that states:

Determine the level of contribution of new information and communication technologies to achieve efficiency of university training Lic Management San Marcos. To achieve the objective we considered the following methodology:

a. First, the unit of analysis for conducting research is the career management of National University of San Marcos is determined.

b. Document analysis of curriculum career and determination of technological platforms.

c. Application of data gathering instruments, to direct users to determine levels of use of new information and communication technologies in vocational training Degree in San Marcos administration, data were processed with SPSS software.

Then he proceeded to analyze the results obtained for the purpose of responding to the problem, through the assumption that the new information and communication technologies contribute to achieving effectiveness of university training of Management Accountant of San Marcos, which finally investigated compared with the objectives that led to its achievement.

Keywords: Information and communication technologies, efficacy, vocational college degree in administration.

\section{INTRODUCCIÓN}

El papel del docente universitario cada vez se hace más complejo dentro del proceso enseñanza-aprendizaje, no solo porque recae en él la responsabilidad de conducir la formación profesional, gestionando las acciones necesarias para alcanzar el logro de las actividades propuestas; sino porque, ante el advenimiento de nuevas tecnologías, tiene que ponerse al tanto, con el fin de adecuarlo, de relacionarlo, mejorar su didáctica y contribuir con el mejoramiento del aprendizaje de sus estudiantes. Se trata, pues, de tener mente abierta y predisposición al cambio, que haga posible aprender a desaprehender para aprehender el nuevo paradigma.

En este sentido podemos decir, que las nuevas tecnologías de información y comunicación (NTICS) están ingresando, progresiva y expansivamente, en el ámbito de los sistemas educativos, no solamente en el nivel básico regular sino fundamentalmente en nivel superior universitario. Las instituciones educativas de nivel superior tienen un rol fundamental en gestionar el acceso a estos nuevos medios, que resultan elementos claves para desenvolverse, acorde a los requerimientos del mercado laboral.
Es necesario considerar que para poder vivir, aprender y trabajar con éxito en una sociedad organizada cada vez más compleja, rica en información y basada en el conocimiento, los estudiantes y los docentes universitarios deben utilizar la tecnología digital con eficacia, con el propósito de lograr una formación profesional cada vez más competente, especialmente en el campo de la administración de negocios, lo que obliga a gestionarla de la manera más eficiente.

La presente investigación muestra una propuesta para afianzar el uso de las NTIC en la eficacia de la formación profesional del Lic. en Administración de la Universidad Nacional Mayor de San Marcos, a partir de los esfuerzos de los docentes y autoridades, referidos a la mejora de la calidad educativa propuesto en el Marco de Acción de Dakar, Educación para Todos: cumplir nuestros compromisos comunes; resaltando en el punto diez lo siguiente: Aprovechar las nuevas tecnologías de información y comunicación para contribuir al logro de los objetivos de la educación para todos.

En la investigación se ha logrado demostrar la contribución de las TIC en pro del logro de los 
objetivos educativos, recomendando la capacitación permanente del docente como mediador del perfil del estudiante en el saber ser, saber hacer, saber conocer y saber estar.

La estructura de la investigación presenta el resumen, la introducción, el marco teórico que sostiene y fundamenta el problema de investigación, el planteamiento del problema, los objetivos, hipótesis, así como el diseño, tipificación de la investigación, sus unidades de análisis, la población y muestra y, finalmente, la metodología; luego se presentaron los resultados obtenidos de la aplicación, análisis y procesamiento de la información; la discusión de los resultados obtenidos, terminando con las conclusiones, recomendaciones y la bibliografía tanto referencial como especializada.

\section{PLANTEAMIENTO DEL PROBLEMA}

Nuevo roles enfrentamos en el tercer milenio que transitamos, con una economía capitalista y globalizada, en la que el cambio en las estructuras y relaciones de la sociedad en su conjunto son fundamentalmente estructurales, y no coyunturales o de momento. Los cambios científico-tecnológicos como factor de competitividad y productividad han cambiado los modos de pensar y de vivir de las organizaciones, lo que ha motivado que las empresas se vean obligadas a transformar sus organizaciones y sus procesos de producción de bienes y servicios, para mantener su vigencia en el mercado.

Observando a nuestro alrededor se puede afirmar, sin temor a equivocarse, que la tecnología ha eliminado una serie de trabajos no calificados, mientras que la necesidad de trabajos mediana y altamente calificados va en aumento. Esto hace reorientar nuestro accionar y tener una visión puesta en el futuro, con un trabajo flexible y un trabajador adaptable a los cambios permanentes y veloces que se presentan; el trabajo real está sustituyendo al trabajo prescrito o tradicional.

Las empresas están reduciendo internamente sus niveles jerárquicos, los procesos se aceleran, las funciones se integran, la departamentalización o fragmentación especializada que generaba "feudos de poder" ha dado paso a la integración y cooperación; consecuencias de la automatización y de la necesidad que tiene el mercado de recibir productos y servicios de calidad en menor tiempo y las empresas se esfuerzan por seguir teniendo presencia en el mercado.
Un nuevo orden económico, social, político y cultural ha surgido en el mundo, que se ha levantado sobre las ruinas y cenizas del anterior; pero, este surgimiento no es producto de la casualidad, sino más bien de una serie de causalidades que debemos conocer para comprender, con una visión de futuro, los retos que debemos enfrentar en el devenir del tiempo.

Muchas fueron las consecuencias de la Segunda Guerra Mundial que impactaron fuertemente en el contexto mundial, entre ellas la referida al surgimiento de la Guerra Fría, que hizo que el mundo se dividiera política, ideológica y económicamente. El orden económico surgido en Occidente alrededor de 1945, como efecto de la revolución industrial, estructuró una producción en serie conocido como modelo de acumulación de capital, fundamentado en el pensamiento y filosofía de Henry Ford, Frederick Taylor, Maynard Keynes; así mismo, se estructuró un sistema de administración que se nutre en el modelo mecanicista de Taylor, que proponía la separación entre la concepción y la ejecución, entre el planeamiento y la acción, entre el saber y el resultado.

Los dos centros de acción geopolítica se enfrentaban por el liderazgo mundial y desarrollaron una carrera armamentista que se realimentaba sustentada en un equilibrio estratégico que, pretendía así, "garantizar la paz mundial".

En cuanto al Perú y al resto de países tercermundistas poseedores de recursos primarios, les correspondió el papel de proveedores de materias primas, alimentos y recursos energéticos.

El proteccionismo de los mercados cedió lugar a su apertura y a un proceso global de desregulación de las economías, sustentadas en "gobiernos democráticos" y garantías a la inversión extranjera y a la libre circulación de capital. El ciclo de desarrollo de las empresas transnacionales y la disponibilidad de recursos financieros producto de la madurez del mercado de capitales hicieron crecer a las empresas y, por lo tanto, a su necesidad de contar con mercados globales. Se impone así un modelo neoliberal y globalizador, cuyas consecuencias las sentimos todos nosotros.

La globalización creó nuevas perspectivas, redefinió el concepto de las economías Estadopaís hacia el concepto de negocio-país. Un nuevo rol del Estado, separado de la propiedad de los medios de producción, más pequeño y eficiente, promotor y garante del nuevo orden económico. 
Las empresas del Estado se privatizan; los capitales adquieren una gran movilidad en un marco donde las comunicaciones, la información, el conocimiento y el capital se globalizan.

El factor humano ha crecido en importancia y hoy es común notar la preocupación de los gobiernos, empresas y trabajadores por formar y capacitar a dicho recurso y que es denominado capital intelectual de un país; es decir, un personal competente y altamente competitivo, que no solamente maneja conocimientos, sino además habilidades, destrezas y actitudes, en otras palabras, competencias profesionales y personales.

La innovación tecnológica, la red de comunicaciones y la expansión y penetración de Internet en las empresas y hogares del mundo, en general y del Perú en particular, están convirtiendo la era de la información y comunicación en la era del conocimiento, en donde el saber y el saber hacer se sitúan como los nuevos paradigmas de la modernidad y a los que el sistema educativo debe asumir para formar hombres libres, para liberar al país de la dependencia y buscar un desarrollo auténtico.

En cuanto a la educación profesional esta llamada tercera revolución, como acontecimiento mundial, requiere, entonces, de una nueva visión de la enseñanza, donde el aprendedor no sea visto como un objeto de la educación, sino como sujeto y actor protagónico de ella. La formación profesional debe hacer uso de la tecnología como medio de facilitar la transmisión y transferencia de "saberes", mas nunca como un fin en sí misma. La formación debe adaptar e innovar sus programas curriculares de formación, de manera que logre vencer el "analfabetismo funcional" que separa al mundo laboral y su demanda, de la oferta de formación profesional, su visión y métodos de enseñanza.

En tal sentido, en la investigación se planteó como problemas de investigación los siguientes:

\section{Problema general:}

¿En qué medida las nuevas tecnologías de información y comunicación contribuyen al logro de la eficacia de la formación profesional universitaria del Lic. en Administración de la UNMSM?

\section{Problemas específicos:}

a. ¿Cuál es el uso de las TIC en la formación profesional del Lic. en Administración de la UNMSM?

b. ¿Cuál es el nivel de pertinencia de la formación profesional del Lic. en Administración de la UNMSM con la demanda laboral?

\section{JUSTIFICACIÓN E IMPORTANCIA DEL ESTUDIO}

La investigación se justifica por el aporte a la formación profesional de calidad y globalizada que garantice al egresado de la Facultad de Ciencias Administrativas insertarse a la vida laboral con rapidez y ocupar cargos que prestigien a la Universidad Nacional Mayor de San Marcos.

Por otro lado, los estudiantes, quienes constituyen nuestro principal usuario, se verán beneficiados en la medida que la enseñanza recibida en la asignatura de NTIC, y en las diferentes asignaturas que conforman el plan de estudios, será fortalecida, a partir del uso intensivo de las NTIC en su formación académica. Y en cuanto a los docentes, se estarán actualizándo permanentemente, de modo que respondan al trabajo que corresponde con dominio de competencias alineadas con estas nuevas tecnologías de información y comunicación. Finalmente, la escuela académico-profesional se verá prestigiada por formar profesionales altamente competitivos que aporten al desarrollo nacional.

\section{HIPÓTESIS PRINCIPAL}

\section{Hipótesis general}

Las nuevas tecnologías de información y comunicación contribuyen al logro de la eficacia de la formación profesional universitaria del Lic. en Administración de la UNMSM.

\section{HIPÓTESIS ESPECÍFICAS}

a. El uso de las TIC en la formación profesional universitaria del Lic. en Administración de la UNMSM es bajo.

b. El nivel de pertinencia de la formación profesional del Lic. en Administración de la UNMSM es mediano respecto a la demanda laboral. 


\begin{tabular}{|l|l|}
\hline \multicolumn{1}{|c|}{ VARIABLES } & \multicolumn{1}{c|}{ INDICADOR } \\
\hline $\begin{array}{l}\text { Las nuevas tecnologías de información } \\
\text { y comunicación }\end{array}$ & $\begin{array}{l}\text { Frecuencia de uso TIC en la formación académica. } \\
\text { Frecuencia de aplicación de TIC. }\end{array}$ \\
\hline $\begin{array}{l}\text { DEPENDIENTE: } \\
\begin{array}{l}\text { Eficacia de la formación profesional uni- } \\
\text { versitaria del Lic. en Administración }\end{array}\end{array}$ & $\begin{array}{l}\text { Las NTIC dinamizan el aprendizaje en aula. } \\
\text { Las NTIC contribuyen en la formación profesional del futuro Lic. en Adminis- } \\
\text { tración. }\end{array}$ \\
\hline
\end{tabular}

Fuente: Propia

\section{METAS ESPECÍFICAS}

1) Diagnosis de la formación del licenciado en Administración en la UNMSM.

2) Determinación del uso de las TIC en la formación académica del futuro egresado de la Escuela Académico Profesional de Administración de la UNMSM.

3) Determinación de necesidades de capacitación docente en el uso de TICs.

\section{MARCO TEÓRICO}

\section{Las TIC y los procesos de enseñanza y aprendizaje}

Disponer de nuevos recursos que puedan permitir nuevas formas de hacer las cosas no significa que necesariamente se produzca el cambio. Antes, el profesor daba sus clases magistrales con el apoyo de la pizarra y los alumnos presentaban sus trabajos y exámenes escritos a mano o máquina; ahora el profesor da sus clases magistrales con Power Point, los estudiantes presentan sus trabajos en Word y a veces los exámenes son pruebas objetivas ante un ordenador. ¿Dónde está el cambio? ¿Innovación o simple comodidad?

La disponibilidad de las TIC por parte de los profesores y de los estudiantes no supone ni mucho menos el fin de los aprendizajes basados en la memorización y la reproducción de los contenidos, ni la consolidación de los planteamientos socio-constructivistas del aprendizaje, a pesar de las magníficas funcionalidades que ofrecen para la expresión personal, la construcción personalizada del conocimiento y el trabajo colaborativo. No obstante, la simple disponibilidad de las TIC sí implica algunos cambios importantes:

- Mayor universalización de la información. El profesor ya no es el gran depositario de los conocimientos relevantes de la materia.

- Metodologías y enfoques crítico-aplicativos para el autoaprendizaje. Ahora el problema de los estudiantes ya no es el acceso a la información (que está casi omnipresente), sino la aplicación de metodologías para su búsqueda inteligente, análisis crítico, selección y aplicación.

- Actualización de los programas. El profesor ya no puede desarrollar un programa obsoleto.

- Trabajo colaborativo. Los estudiantes se pueden ayudar más entre ellos y elaborar trabajos conjuntos con más facilidad a través del correo electrónico, los chats, entre otros.

- Construcción personalizada de aprendizajes significativos.

\section{LA FORMACIÓN DEL PROFESORADO EN TIC}

Las TIC no solamente suponen más tiempo de dedicación para el profesorado, sino que también traen consigo nuevas necesidades de formación, que a su vez van a exigir nuevas inversiones de tiempo. Esta nueva formación relacionada con las TIC que requiere el profesorado universitario se centra en los siguientes aspectos: 
$\checkmark$ El uso de los aparatos y programas informáticos de uso general: entorno windows, procesador de textos, navegador de Internet y correo electrónico.

$\checkmark$ El conocimiento de las funcionalidades que ofrece el "campus virtual" de la propia universidad. La aplicación de las TIC a la enseñanza como instrumento de innovación didáctica: creación de la página web de la asignatura, organización de la tutoría virtual con sus alumnos, aprovechamiento de los recursos de Internet para las clases y para proponer actividades a los estudiantes.

$\checkmark$ Conocimiento y utilización de las bases de datos y programas informáticos específicos de la materia que se imparte (instrumento profesional).

$\checkmark$ Elaboración de páginas web de interés relacionadas con la materia.

La mejor manera de lograr esta nueva capacitación del profesorado en TIC es promoviendo la adecuada formación desde la propia universidad, incentivando el uso y la integración de las TIC y, por supuesto, facilitando los adecuados medios tecnológicos y un buen asesoramiento continuo.

\section{EL IMPACTO DE LAS TIC EN EL APRENDIZAJE ${ }^{1}$}

Sustentado en la teoría de Vygotsky, cuya primera razón es la de destacar que el ser humano solo puede aprender o desarrollarse (términos que para Vygotsky se refieren a dos fases de un mismo proceso inter - intra) y llegar a funciones de nivel superior en la medida en que haya una mediación cultural en la que exista una interacción tanto con otros agentes sociales como con otras herramientas culturales.

La segunda razón es que la propuesta vigotskiana, junto a este énfasis en el origen social del conocimiento, destaca también que el conocimiento se construye porque hay una mediación semiótica.

Las características señaladas son las siguientes: formalismo, interactividad, dinamismo, multimedia, hipermedia, conectividad.
Todas estas características tienen, como vemos, la potencialidad de modificar los procesos de gestión del conocimiento y, a nuestro juicio, contribuirían fundamentalmente a tres aspectos esenciales en los procesos de enseñanza y aprendizaje.

\section{COMPETENCIAS EN TECNOLOGÍAS DE INFORMACIÓN Y COMUNICACIÓN (TIC) ${ }^{2}$}

No se trata de establecer con las competencias TIC un nuevo currículo o una nueva área de conocimientos en la educación obligatoria. Consideramos las competencias en TIC como un instrumento que ayuda a lograr los objetivos del currículo ordinario en los aspectos conceptuales, actitudinales y procedimentales. Pero además es necesario trasladar a nuestros alumnado un conjunto de habilidades y destrezas TIC necesarias socialmente, eso que en ocasiones se llama nueva alfabetización.

\section{LAS CARACTERÍSTICAS DE LAS TIC Y SUS POSIBILIDADES EDUCATIVAS}

De forma incluso no planificada, las TIC se utilizan como instrumentos en la enseñanza y el aprendizaje, tanto por parte del profesorado, como por parte del estudiante, fundamentalmente en cuanto a la presentación y búsqueda de información. Más allá, podemos hablar de que las TIC pueden suponer un salto mayor si se explotan sus potencialidades de forma más profunda, imaginativa y coherente, de acuerdo con las posibilidades que permiten. Como dice Judit Minian:

Pensar informáticamente supone operaciones mentales distintas y, por lo tanto, una propuesta pedagógica específica. No se puede pensar que el poder de la tecnología por sí solo va a conseguir que los viejos procesos funcionen mejor. Su uso debe servir para que las organizaciones sean capaces de romper los viejos moldes y creen nuevas formas de trabajo y funcionamiento.

\section{LAS NUEVAS TECNOLOGÍAS DE INFORMACIÓN Y COMUNICACIÓN (NTIC) EN LA GESTIÓN}

Un modo de mejorar el funcionamiento de los centros por aplicación de las TIC es introduciendo

1 Elena Martín, Las TIC: del aula a la agenda política. Ponencias del Seminario internacional Cómo las TIC transforman las escuelas, 2008. UNICEF ARGENTINA, $55-70$.

2 UNESCO. Estándares de competencias en tic para docentes. Londres, Enero 8 de 2008. Normas sobre Competencias en TIC para Docentes, $11-17$. 
soluciones para mejorar la gestión de estos. Las TIC son poderosas herramientas para procesar la información, que se adaptan especialmente bien a este propósito. Parte de la responsabilidad de este uso de las Nuevas Tecnologías de Información y Comunicación (NTICS) en la gestión la tiene la Administración, que ha implementado vías de comunicación entre los centros y ella a través de las NTIC (concretamente, a través de aplicaciones web). No obstante, hay muchos otros aspectos en los que las Nuevas Tecnologías de Información y Comunicación (NTIC) pueden suponer una mejora eficaz de los procesos de gestión.

\section{EFICACIA}

Siempre ha habido cierta preocupación por identificar los rasgos que caracterizan a las escuelas eficaces o escuelas con éxito. La visión clásica de este problema plantea que la calidad de un centro depende, fundamentalmente, de sus elementos personales, es decir, de sus profesores y alumnos. Las escuelas eficaces son aquellas que tienen buenos profesores y buenos alumnos y donde, por tanto, cabe esperar excelentes rendimientos. Se ha demostrado que esta suposición -aunque parte de un principio que inicialmente es cierto -es inexacta, ya que en escuelas con parecidos recursos humanos se obtienen los mismos o idénticos resultados.

\section{FORMACIÓN PROFESIONAL}

Los países en desarrollo enfrentan una serie de retos no solo por las características y precariedad de sus propios sistemas económicos y sociales, sino también por las consecuencias de los ritmos y cambios en la sociedad internacional. Es bien sabido que existe un nuevo ordenamiento socioeconómico en el mundo producido por el proceso de globalización y flexibilización de la economía. El mundo se encoge, pues la tecnología de las comunicaciones permite no solo un incremento de la información sino que esta sea accesible a todos y se comparta rápidamente. Todo ello ha cambiado las relaciones entre los países, las empresas (dentro de y entre ellas), los clientes, los proveedores y sus entornos.

En este contexto, la formación, sobre todo la dirigida a la reconversión profesional de los trabajadores, asume una importancia creciente como una medida eficaz para facilitar la adecuación de las capacidades de los trabajadores a los requisitos del nuevo contexto laboral, así como para minimizar las fricciones e inconveniencias causadas por estos ajustes en el ámbito productivo y entre los trabajadores.

\section{RELACIONES ENTRE EL PERFIL PROFESIONAL Y LOS OBJETIVOS FORMATIVOS DEL PROFESIONAL UNIVERSITARIO}

Si se define el perfil profesional como un conjunto de características, conductas o funciones que se estima deberá tener o ser capaz de exteriorizar con eficiencia y eficacia quien egrese como profesional, es obvio entonces que esta idea de "producto", será la que sirva de base para la elaboración de los "objetivos educativos formativos" de dicho profesional. Esto significa que existe una estrecha relación entre el perfil profesional y los objetivos educativos formativos del profesional que se quiere formar.

\section{ESTRUCTURA CURRICULAR GLOBAL EN LA FORMACIÓN DE PROFESIONALES}

La estructura curricular global es entendida como el conjunto de elementos o componentes curriculares que han sido considerados para la formación de un profesional. Estos elementos o componentes considerados en la estructura curricular global responden a los objetivos educativos $y$, por ende, al perfil profesional y su determinación depende de cómo se ha decidido la formación del profesional.

Estos dos grandes componentes de la estructura curricular global (formación general y formación estrictamente profesional) pueden subdividirse en: ${ }^{3}$

\section{1 . Formación general:}

a) Formación científica básica, comprende aquellos bloques de asignaturas de las ciencias formales y ciencias fácticas básicas.

b) Formación humanística, comprende disciplinas de carácter no científico que son requeridas por los objetivos formativos, tales como lenguaje, filosofía, etc.

c) Formación institucional, comprende asignaturas o proyectos que pudieran diseñarse y a través de los cuales se transmitiría la

\footnotetext{
3 Flores Barboza, José. Apuntes de Clase. Asignatura. Currículum Universitario.1985. Maestría Administración Universitaria - UNMSM.
} 
particular concepción de la facultad respecto a lo que debe ser el egresado como profesional, ciudadano y persona (aspectos formativos complementarios).

\section{Formación profesional:}

a) Formación profesional básica, aquella formación común o formación en aspectos generales de la profesión, conformada por aquellos bloques de asignaturas que permitirían el logro de los objetivos educativos y de los rasgos del perfil profesional referidos a las conductas, comportamientos o funciones que son comunes a todos los que siguen una profesión, al margen de las especializaciones que deseen hacer.

b) Formación especializada, comprende las asignaturas de especialización en alguna rama o campo profesional.

c) Práctica pre-profesional, que deberá garantizar al futuro profesional no solo adquirir las habilidades en las funciones que desempeñará en su vida profesional, sino las destrezas necesarias sobre estas funciones.

Es necesario precisar que en la formulación específica de las asignaturas que forman parte de cada componente se considera otras variables como el tiempo, que puede hacerse en función de porcentajes a fin de poder precisar la cantidad de horas por cada componente, así como las de cada asignatura. Elías J. Rossi Quiroz ${ }^{4}$, señala en forma referencial, por ejemplo, para la formación general $15 \%$, formación científica $7 \%$, formación humanística $6 \%$, formación institucional $2 \%$; formación profesional $85 \%$, formación básica $50 \%$, formación especializada $20 \%$ y formación preprofesional $15 \%$.

\section{LA ADMINISTRACIÓN Y LA PROFESIÓN DEL LICENCIADO EN ADMINISTRACIÓN}

Abraham Maslow dice que para poder satisfacer las necesidades debemos empezar por nosotros mismos y después en grupo.

Por su parte, Fremont E. Kast dice que la administración es la coordinación de hombres y recursos materiales para el logro de objetivos organizados, lo que se logra por medio de elementos:

1. dirección de objetivos
2. a través de gente

3. dentro de una organización

\section{ROLES DEL ADMINISTRADOR}

La realidad es que los principios y técnicas administrativas modernas deben aplicarse al uso racional de los recursos para el logro eficiente de resultados, por lo que al administrador le corresponde contribuir, valiéndose de sus conocimientos profesionales, a que el organismo en que presta sus servicios, público o privado, lucrativo o no, alcance sus metas en forma eficiente, mediante estructuración y coordinación adecuadas de los recursos disponibles.

Las funciones del administrador varían de acuerdo con la posición (nivel jerárquico) que ocupa en la estructura organizativa.

\section{ÉTICA PROFESIONAL}

La ética se puede definir como el punto de vista de las cuestiones morales que busca un individuo; sobre todo, una respuesta, una orientación práctica respecto a los problemas concretos de la sociedad, y por eso incluye las cuestiones que se manifiestan en la vida política, económica y social.

La ética estudia la conducta humana, sus normas, derechos y deberes con respecto a la sociedad, en que se desarrolla, y señala lo que "debe" o "no debe" hacerse en una agrupación social determinada; es decir, la ética es el comportamiento del hombre profesionista ante su propia sociedad, formado de aptitudes y valores que lo caracterizan como tal, con un comportamiento adecuado, siempre en busca del bien, la verdad y, sobre todo, la rectitud.

\section{METODOLOGÍA Y TÉCNICAS DE INVESTIGACIÓN}

\section{NIVEL DE INVESTIGACIÓN}

La investigación responde a una de tipo aplicada y el nivel es exploratorio, evaluativa y ex post facto.

\section{POBLACIÓN Y MUESTRA POBLACIÓN}

La población seleccionada para el presente estudio estuvo constituida por la población estudiantil de la Escuela Académico Profesional de

4 Elias J. Rossi Quiroz. Ibidem, pag. 25. 
Administración de la Facultad de Ciencias Administrativas de la UNMSM, como se observa en el cuadro No 1, que a continuación se indica:

CUADRO N ${ }^{\circ} 1$

ALUMNOS MATRICULADOS - ADM POR BASE

\begin{tabular}{|c|c|}
\hline AÑO DE INGRESO (BASE) & $\begin{array}{c}\text { TOTAL X AÑO DE } \\
\text { INGRESO }\end{array}$ \\
\hline 2010 & 218 \\
\hline 2006 & 216 \\
\hline 2008 & 215 \\
\hline 2009 & 206 \\
\hline 2007 & 200 \\
\hline TOTAL MATRICULADOS & 1055 \\
\hline
\end{tabular}

Fuente: Elaboración propia.

Como puede observarse en el cuadro No 1, la población de estudio $(\mathrm{N})$ es:

$\mathrm{N}=1,055$

Esta es una población finita dado que:

$\mathrm{N}<100,000$

MUESTRA:

La muestra se determinó con la fórmula siguiente:

$\mathrm{n}=\left(\mathrm{Z} 2 * \mathrm{p}^{*} \mathrm{q} * \mathrm{~N}\right) /(\mathrm{e} 2(\mathrm{~N}-1)+(\mathrm{Z} 2 * \mathrm{p} * \mathrm{q})) \ldots(1)$

Donde:

n: tamaño de la muestra.

e: margen de error.

Z: margen de confiabilidad y se obtiene de tablas:

\begin{tabular}{|c|c|}
\hline $95 \%$ & $99 \%$ \\
\hline$Z=1.96$ & $Z=2.58$ \\
\hline$a=0.05$ & $a=0.01$ \\
\hline
\end{tabular}

$\mathrm{p}=$ Frecuencia esperada del factor a estudiar.

$\mathrm{q}=1-\mathrm{p}$

Reemplazando valores en (1), tenemos:

$\mathrm{n}=281.81$

Entonces, debemos encuestar a 282 estudiantes.

\section{RECOLECCIÓN E INTERPRETACIÓN DE DATOS}

La metodología empleada se detalla a continuación:

a. En primer lugar, se determinó la unidad de análisis, que estuvo conformada por la población estudiantil de la Escuela Académico Profesional de Administración de la Facultad de Ciencias Administrativas de la Universidad Nacional Mayor de San Marcos. Se le aplicó la encuesta que tuvo como objetivo explorar sus necesidades de uso de TIC y la percepción sobre su formación profesional.

b. Análisis de la diagnosis realizada y de la malla curricular de la carrera profesional de Administración de la Facultad de Ciencias Administrativas, como se indica:

\section{Programa de capacitación docente (módulos)}

Personal docente de la Facultad de ciencias administrativas

\begin{tabular}{|c|c|}
\hline Docentes nombrados & 111 \\
\hline Docentes contratados & 72 \\
\hline Total Docentes & 183 \\
\hline
\end{tabular}

Fuente: Unidad de Estadística e Informática de la UNMSM. 2008.

\section{Metodología}

La ejecución de estos módulos será destinada a toda la plana docente de la facultad, ya que si bien existe un número determinado de docentes por escuela, este es aparente, es decir, muchas veces, se hacen "préstamos de personal" entre las 3 escuelas. Por tanto, no se sabe a ciencia cierta un número real de docentes por cada una de ellas.

Según las estadísticas, existen un promedio de 183 docentes en la facultad. Las capacitaciones a llevarse a cabo tendrán como principal instrumento: los equipos de cómputo. En la facultad existen 183 computadoras para uso académico, gracias a una renovación realizada el año 2008.

Equipos de cómputo

\begin{tabular}{|c|c|}
\hline Área administrativa & 59 \\
\hline Área académica & 183 \\
\hline Total computadoras operativas & 242 \\
\hline
\end{tabular}

Fuente: Unidad de Estadística e Informática de la UNMSM. 2008.

Además se observó la existencia de 4 laboratorios disponibles para usos académicos, por tanto la ejecución de cada módulo se llevará a cabo en ellos.

Los módulos 0 y 1 se llevarán a cabo con todos los docentes, en 2 ciclos de 2 semanas y 2 meses respectivamente, durante el periodo de vacaciones académicas. 
El primer ciclo tendrá lugar en los meses de enero-marzo y el segundo ciclo, de julio-setiembre. Los docentes tendrán opción de llevar la capacitación en cualquiera de estos dos periodos, cada ciclo tendrá un cupo igual a la mitad del total de docentes de la facultad, con asistencia obligatoria, salvo motivos excepcionales o de fuerza mayor.

El módulo 2 se llevará a cabo en el ciclo de vacaciones siguiente al ciclo en el que se llevaron los módulos anteriores. Aquellos docentes que llevaron el módulo 0 y 1 en el ciclo de enero-marzo, deberán llevar el siguiente módulo en el ciclo de julio-setiembre.

Para la ejecución del módulo 2, se agruparán a los docentes según especialidad y tendrá una duración de 6 meses.

Estos 3 primeros módulos en conjunto se implementarán durante el primer año de la ejecución del proyecto. El módulo 3, se implementará en caso de adquisición de nuevos equipos de cómputo o audiovisuales o la implementación de nuevos softwares actualizados para uso de los docentes o de la especialidad de la carrera, por tanto su aplicación será esporádica. Dentro del horizonte del proyecto, en promedio se implementarán 2 capacitaciones de este módulo. Su duración dependerá del motivo de su aplicación.

Al finalizar cada módulo se llevará a cabo una evaluación final para determinar la efectividad del programa. Así mismo dentro de cada módulo se realizarán evaluaciones prácticas constantes a los beneficiarios.

El módulo 3 también será aplicado en caso se determine, previa evaluación, algunas falencias en los conocimientos adquiridos en los módulos predecesores.

\section{CONTENIDO DE LA CAPACITACIÓN}

El proceso de capacitación constará de 4 módulos:

MÓDULO 0: Actualización en cuanto al uso y manejo de la infraestructura tecnológica de la facultad.

Objetivo: Introducir al docente en el manejo de la infraestructura tecnológica con la que cuenta la facultad.

Se busca que el docente tenga conocimientos sobre cuáles son los equipos existentes y reconozca sus principales componentes, características y usos. La capacitación se llevará a cabo in situ, en los laboratorios con los que cuenta la facultad, así como en los salones en los que se dictan las clases. Se dará prioridad a la enseñanza de los siguientes hardwares: computadoras, láptops, proyectores, ecrans, parlantes y otros que se crean convenientes. Además se describirán brevemente, a modo de introducción, las características y usos de intranet, el catálogo del sistema de bibliotecas (SISBIB), tanto de la facultad como de la biblioteca central, y el aula virtual.

\section{Duración: $\quad 2$ semanas.}

Periodicidad: Diario.

Alcance: Todos los docentes de la Facultad de Administración (183).

Modalidad: Como una extensión de los servicios del Centro de Informática de la Universidad.

MÓDULO 1: Manejo de Windows 7, Microsoft Office 2010, Internet.

Objetivo: Reconocer los usos, funciones y características del sistema operativo WINDOWS 7 , el paquete de procesamiento de datos Microsoft Office 2010, Internet y herramientas virtuales usadas en la facultad.

Se busca el reconocimiento del espacio de trabajo y compatibilidad con versiones anteriores del sistema operativo y el paquete de software de procesamiento de datos (Word, Excel, Power Point), además de instruirlo en el manejo de Internet y sus principales motores de búsqueda, correo electrónico, y de las herramientas usadas por la facultad: aula virtual, intranet, catálogo en línea y software de búsqueda de libros de la biblioteca. Los docentes prepararán sus clases a modo de práctica, utilizando estas herramientas a lo largo de la duración del módulo.

$\begin{array}{ll}\text { Duración: } & 2 \text { meses. } \\ \text { Periodicidad: } & \text { Diario. }\end{array}$

Alcance: Todos los docentes de la Facultad de Administración (183).

Modalidad: Como una extensión de los servicios del Centro de Informática de la Universidad.

MÓDULO 2: Manejo y aplicación de software según la especialización del docente.

Objetivo: Capacitar al docente en el manejo de software según su especialidad. 
Se busca formar un docente competitivo en su área de especialización, por medio de software de finanzas, contabilidad, marketing, logística, recursos humanos, estadística, entre otros. Para la ejecución de este módulo, se recurrirá a convenios con instituciones que brinden cursos o talleres en las especialidades mencionadas.

\section{Duración: \\ 6 meses. \\ Periodicidad: 2 veces por semana.}

Alcance: Se agrupará a los docentes según especialidad.

Modalidad: Por convenio con instituciones/ Facultades dedicadas a brindar asesoría o capacitación en las áreas de especialización de la carrera.

MÓDULO 3: Actualización docente en el manejo y aplicación de software según especialidad.

Objetivo: Actualizar al docente en el manejo y aplicación de las últimas versiones de los softwares de su especialidad.

Por medio de este módulo se capacitará al docente sobre algunas nuevas características o funciones de versiones más actualizadas de los softwares que utiliza para desarrollar su curso. Además también se ejecutará en caso de la adquisición de nuevos equipos de cómputo o audiovisuales en general. A modo de actualización, se implementará de forma anual.

Duración:

Entre 2 semanas y 1 mes.

Periodicidad: Interdiario.

Modalidad: Por convenio con instituciones/ facultades dedicadas a brindar asesoría o capacitación en las áreas de especialización de la carrera.

En relación con la enseñanza del manejo de softwares especializados de carrera, presenta dificultades ya que los docentes tampoco cuentan con los conocimientos necesarios sobre ellos. Con este diagnóstico se busca ponerle fin a esa carencia dentro de la formación académica en la Escuela Académico Profesional de Administración. Para la atención a este problema se determinó trabajarlo en 3 etapas:

PRIMERA ETAPA: Mejora y perfeccionamiento de la sumilla del curso de Nuevas Tecnologías de la Información y las Comunicaciones.

SEGUNDA ETAPA: Implementación de la enseñanza del manejo de software en las horas de práctica de los cursos de la malla curricular.
Para esta etapa se llevará a cabo la realización de todo un plan de enseñanza que integre softwares especializados, ya sea por área o por curso, dentro de las horas de práctica disponibles en la actual malla curricular.

Esta etapa tendrá dos fases:

- De $1^{\circ}$ a $4^{\circ}$ ciclo, se enseñarán softwares de cursos básicos como Estadística o Matemática, y la introducción a software especializados por medio del curso de NTIC.

- De $5^{\circ}$ a $10^{\circ}$ ciclo, la enseñanza se centrará en los cursos de carrera y su respectivo software según área de especialidad, como Finanzas, Recursos Humanos, Marketing, Logística, etc. En el décimo ciclo se implementarán también la enseñanza de software de Gerencia y Alta Dirección.

Requerimiento de software a implementarse en las horas de práctica de los cursos de la malla curricular

\begin{tabular}{|l|l|}
\hline \multicolumn{1}{|c|}{ ÁREA } & \multicolumn{1}{c|}{ SOFTWARE } \\
\hline Matemática & $\checkmark$ Macros de Excel - SOLVER \\
\hline & $\checkmark$ SPSS \\
Estadística & $\checkmark$ MINITAB \\
\hline Marketing & $\checkmark$ TORA \\
\hline & $\checkmark$ ORACLE \\
& $\checkmark$ BMART BUSINESS APLICATION \\
Gestión & $\checkmark$ SAP BUSINESS ONE \\
& $\checkmark$ MICROSOFT DYNAMICS \\
& $\checkmark$ ADMINPAQ \\
\hline Formulación y & $\checkmark$ PUSINESS MODELER ADVANCED \\
evaluación de & $\checkmark$ Visio \\
proyectos & $\checkmark$ Crystalball \\
\hline & $\checkmark$ Visio \\
Logística & $\checkmark$ BSC \\
& $\checkmark$ SISTEMATIC \\
\hline Legislación & $\checkmark$ WORKFLOW \\
tributaria & $\checkmark$ PDT \\
\hline
\end{tabular}

Fuente: Elaboración propia.

TERCERA ETAPA: Seguimiento de egresados y evaluación de su desempeño en el manejo de TIC en su respectivo centro de trabajo.

a. Análisis de la malla curricular de estudio de las carreras profesionales motivo de investigación (anexo 3). 
b. Análisis bibliográfico.

c. Aplicación de instrumentos de recogimiento de datos para determinar niveles de percepción del empleo de las nuevas tecnologías de información y comunicación en la formación profesional.

d. Aplicación de software para el relevamiento y tratamiento estadístico.

\section{TÉCNICAS DE PROCESAMIENTO Y ANÁLISIS DE DATOS}

Las técnicas utilizadas para el procesamiento y análisis de datos obtenidos en la encuesta son:

1. Tabulación.- Para expresar los datos de estudio valiéndose de tablas estadísticas, la cantidad de datos organizados fueron trabajados con el programa estadístico para ciencias sociales (SPSS Vs. 21).

2. Tablas analíticas o de resumen.- Para la presentación de una relación de resultados expresada mediante un esquema numérico, con el objeto de facilitar al lector la interpretación.

3. Se utilizó el SPSS en su versión 21, para determinar parámetros de estadística descriptiva (frecuencias, medias, modas, desviación estándar, etc.) y correlaciones para prueba de hipótesis.

\section{DISCUSIÓN DE RESULTADOS}

A la luz de los resultados estadísticos obtenidos del análisis de la información extraída de la aplicación de las encuestas a los estudiantes de la Escuela Académico-Profesional de Administración de la FCA - UNMSM, se determinó que:

1. El $81.56 \%$ de los estudiantes encuestados manifestaron que no se aplica el uso de las NTIC durante el desarrollo de clases por parte de los docentes.

2. El $69.86 \%$ mostró su preocupación sobre la necesidad del uso frecuente de las NTIC por parte de los docentes en sus asignaturas que desarrollan.

3. El $59.22 \%$ manifestó la dificultad de uso de las NTICs en la realización de sus trabajos académicos formativos.

4. El $31.56 \%$ de los estudiantes calificaron a los docentes que no tienen las competencias necesarias en el uso de las NTIC.
5. El $97.16 \%$ de los encuestados manifestaron la necesidad de dar mayor énfasis en la aplicación de las TIC en su formación académica.

6. El $68.12 \%$, en promedio, opinó que las NTIC contribuyen de manera dinámica y participativa en el proceso de aprendizaje.

7. El $87.3 \%$ en promedio manifestó que las NTIC favorecen al aprendizaje de los contenidos en sus diferentes asignaturas.

8. El $84.8 \%$ de los estudiantes respondieron que el uso de las TIC favorecen mayor cantidad de contenidos.

9. El $60.28 \%$ respondió que las herramientas de NTIC son de calidad.

10. El $99.3 \%$ de los estudiantes encuestados respondieron que la aplicación de las NTIC contribuyen en la formación profesional del futuro licenciado en Administración.

Dado que la investigación es aplicada y de tipo exploratorio, evaluativo y ex post facto, se ha demostrado, con los hallazgos fruto de la observación y análisis de los resultados del trabajo de campo, que el uso de las NTIC contribuyen en el logro de la eficacia de la formación profesional universitaria del licenciado en Administración de la UNMSM y que es percibido con mucha incidencia por parte de los usuarios directos de los servicios educativos, que son los estudiantes de la Facultad de Ciencias Administrativas. En consecuencia, al demostrarse el logro de los objetivos, los problemas planteados en forma de preguntas son solucionados y demostrados con las respuestas positivas planteadas hipotéticamente.

\section{CONCLUSIONES}

1. Los docentes de la Escuela Académico Profesional de Administración de la Facultad de Ciencias Administrativas de la UNMSM, actualmente, deben adquirir nuevas estrategias de enseñanzas, las que les permitirán desarrollar capacidades y habilidades en sus alumnos, para lo cual es fundamental la capacitación en el uso de las nuevas tecnologías de información y comunicación. Si un profesor logra desarrollar las competencias para el uso de las TIC, no solo le permitirá mejorar su labor docente, sino que también el ámbito en donde se desempeñe, ya que al modificar ciertas estrategias de enseñanza-aprendizaje, permite innovar el currículo, a partir de un nuevo perfil 
profesional tanto del ingresante, egresado como del docente, generando cambios, en la escuela profesional, orientando a crear una cultura de autoevaluación y mejora continua con fines de acreditación.

2. Si bien existen iniciativas a nivel de la Escuela Académico Profesional de Administración, sobre capacitación docente en cuanto a uso y aplicación de las NTIC, se debe desarrollar un serio compromiso por parte de la gestión con programas estructurados dentro del planeamiento educativo y orientado por el modelo educativo correspondiente a la escuela, alineado con el modelo educativo de la UNMSM que fundamente el nuevo perfil del egresado de la Escuela Académico Profesional de Administración, a fin de fortalecer las capacidades y competencias de los profesionales, de manera que se asegure niveles adecuados de empleabilidad en el mercado laboral.

3. La presente investigación abre la posibilidad de superar esta debilidad de la Escuela Académico Profesional de Administración-UNMSM, con el propósito principal de hacer notar la importancia que reviste el uso de las NTIC en la formación profesional de modo que hagan posible desarrollar nuevas competencias en el dominio y uso de las NTIC en el estudiante y le permita obtener un alto nivel competitivo en el mercado laboral.

\section{RECOMENDACIONES}

La Universidad Nacional Mayor de San Marcos, decana de América, es una universidad estatal de prestigio, no solo por sus años de experiencia y formación académica profesional, sino también por la alta capacidad de experiencia y conocimiento por parte de sus docentes y por la metodología que aplica en sus exámenes de ingreso, con lo cual exige a los postulantes tener el perfil de un estudiante con dominios en la diversas materias. Por ello, se recomienda a esta prestigiosa universidad, y en particular a la Escuela Académico Profesional de Administración, poner mucho énfasis en lo que respecta a los requerimientos de las NTIC en la formación profesional del estudiante de esta unidad académica, durante todo el proceso de formación y de esta manera este pueda obtener beneficios acordes con los requerimientos del mercado laboral.

Así mismo, la selección de personal docente debe realizarse en función conocimientos en el uso de TIC, así como también recurrir a las capacitaciones en actualización de software por parte de los profesores estables y contratados, en las diferentes especialidades de la carrera de Administración.

Es de esperar que a partir de la presente investigación se inicie un nuevo trabajo en la gestión de la carrera de Administración bajo un sistema de gestión de la calidad educativa, a fin de potenciar seriamente la formación académica, de manera que sus profesionales egresados salgan con las competencias que el mercado laboral y la sociedad en su conjunto demanda de profesionales altamente competitivos y con conciencia social, comprometidos con el desarrollo cultural, social y económico del país.

\section{REFERENCIAS BIBLIOGRÁFICAS}

Martín, Elena (2008). Las TIC: del aula a la agenda política. Ponencias del Seminario internacional Cómo las TIC transforman las escuelas, 2008. UNICEF ARGENTINA, 55 - 70.

UNESCO (2008). Estándares de competencias en tic para docentes. Londres, Enero 8. Normas sobre Competencias en TIC para Docentes, $11-17$.

Echevarria, Javier (2000). Tercer Entorno (3). 2000b, Edit. Monteso, p 45.

Area, Manuel y García Valcárcel, Ana (2001). Materiales Didácticos Digitales. 2001, edit. Limusa, p 89.

Choque Larrauri, Raúl (2009). Estudio de aulas de innovación pedagógica y desarrollo de capacidades TIC (Tesis para optar el grado académico de Doctor en Educación), UNMSM, 2009.

Ministerio de Educación (2009). Diseño Curricular Nacional de Educación Básica Regular. Lima: MINEDU, 2009.

Ministerio del Poder Popular para la Educación. (2008) Manual de consulta.

Módulo I. Las Tecnologías de la Información y la Comunicación. Venezuela, 2008.

Cabrero, Julio (2002). Entornos de Formación Telemáticos. 2002, edit Limusa, p 105.

Minian, Judit. Uso de las Tecnologías. 1999. P 67-74.

Rossi Quiroz, Elías. (1993). El Perfil Profesional y la Planificación Curricular a Nivel Universitario: Lima: Edic. ER, 1993. 
Mira y López, Emilio (1965). Manual de Orientación Profesional. Buenos Aires, Edit. Kapelusz, 1965, (6º Edición).

Mager, Robert - Beach, Kenneth (1972). Enseñanza de los Oficios en la Escuela Vocacional. México, AID, Edit. PAX, 1972.

Gagne, Robert - Briggs, Lesli (1976). La Planificación de la Enseñanza. México, Edit. Trillas. ( $1^{\circ}$ Edic. en español).

Ministerio de Educación (INIDE-COTEC) (1976). Estudios Básicos del Curriculum en el Sistema Educativo Peruano. Lima, INIDE.

Ministerio de Educación, Dirección General de Educación Superior (1976). Estructura Curricular del I Ciclo de Educación Superior. Lima, 1976.

Ministerio de Educación (DIGES). (1982) Procedimientos para la Elaboración de Perfiles Profesionales. Lima , 1982.

Campos Arenas, Agustín (1986). Metodología para la Elaboración del Perfil Genérico: Lima, en Revista Perspectivas de la Educación, № 3 , Mayo 1986.

Rossi Quiroz, Elias J: Ibidem: 13.

Kaufman, Roger A. (1983). Planificación de Sistemas Educativos. Ideas Básicas Concretas. México, Edit. Trillas, 1983, (9º reimpresión).

Flores Barboza, José (1985). Apuntes de Clase. Asignatura. Curriculum Universitario.1985. Maestría Administración Universitaria - UNMSM.

\section{REFERENCIAS ELECTRÓNICAS}

http://www.unmsm.edu.pe/transparencia/archivos/Plan_E_Institucional_2007_2011_ mod22_08_08.pdf.

http://www.unmsm.edu.pe/ogpl/estadistica/

http://www.unmsm.edu.pe/occaa/publicaciones/ normatividad.pdf

http://sisbib.unmsm.edu.pe/BibVirtual/Publicaciones/Inv_Educativa/2010_n25/pdf/ a12v14n25.pdf

http://www.unmsm.edu.pe/transparencia/archivos/Plan_E_Institucional_2007_2011_ mod22_08_08.pdf

http://fundacionsanmarcos.blogspot.com/2010/11/ impacto-social-de-la-incorporacion-de.html

http://www.unmsm.edu.pe/Noticias2007/julio/d16/ veramp.php?val = 1

http://peremarques.pangea.org/ticuniv.htm

http://www.monografias.com/trabajos30/licenciado-administracion/licenciado-administracion.shtml\#ixzz2xpq9BhMH.

Rosario, Jimmy. “TIC: Su uso como Herramienta para el Fortalecimiento y el Desarrollo de la Educación Virtual”. Disponible en el ARCHIVO del Observatorio para la Ciber Sociedad en http://www.cibersociedad.net/archivo/ articulo.php?art=221, 2006. 